\title{
Severe Japanese Spotted Fever Successfully Treated with Fluoroquinolone
}

\author{
Masafumi Seki ${ }^{1}$, Naruhiko Ikari ${ }^{2}$, Seigo Yamamoto ${ }^{3}$, Yuhki Yamagata ${ }^{2}$, Kosuke Kosai ${ }^{1}$, \\ Katsunori Yanagihara ${ }^{1}$, Tomoyuki Kakugawa ${ }^{2}$, Shintaro Kurihara ${ }^{1}$, Koichi Izumikawa ${ }^{1}$, \\ Yoshitsugu Miyazaki ${ }^{1}$, Yasuhito Higashiyama ${ }^{1}$, Yoichi Hirakata ${ }^{1}$, \\ Takayoshi Tashiro ${ }^{1}$ and Shigeru Kohno ${ }^{1}$
}

\begin{abstract}
A 77 years old woman who had a bite with eschar on her left arm, was admitted to emergency ward in our hospital, because of high fever, severe malaise, skin eruption, and consciousness disturbance beginning 5 days previously. She was diagnosed as Japanese spotted fever by seropositive of Rickettsia japonica (R. japonica) antibody, and successfully treated with fluoroquinolone, after minocycline hydrochloride had been proven ineffective. $R$. japonica-specific DNA was detected by PCR from the tick: Haemaphysalis hystricis larvae collected from a mountainous location in Fukuoka, Japan where the patient had been bitten.
\end{abstract}

Key words: Japanese spotted fever, Rickettsia japonica, fluoroquinolone, tick, Haemaphysalis hystricis

(DOI: 10.2169/internalmedicine.45.1831)

\section{Introduction}

The first case of Japanese spotted fever caused by Rickettsia japonica ( $R$. japonica) was reported in Japan in 1984. For several days after1984, severe cases have been sporadically reported, however, most cases were cured by tetracyclines (1-3). Kodama et al. reported twenty-eight patients with Japanese spotted fever were treated with minocycline, and all recovered, excluding one patient with a severe course (2). We encountered a patient with severe Japanese spotted fever who was not cured by tetracycline, but treated successfully with fluoroquinolone.

\section{Case Report}

A 77-year-old woman who was generally in good health complained of a sudden overall weakness in early May, 2005 (Clinical Day 0), after collecting bamboo shoots from a mountain in Munakata City, Fukuoka Prefecture, Japan.
She was admitted to Yahata Hospital, Kitakyushu City (Fukuoka Prefecture) 5 days later (Clinical Day 5) with a fever of $39.3^{\circ} \mathrm{C}$, systemic erythema and purpura, and a consciousness level of 13 (E3V4M6) on the Glasgow Coma Scale. Blood pressure was $144 / 74 \mathrm{mmHg}$ and the pulse rate was 88 beats/min. Cyanosis, cardiac murmur and breath sounds were absent, and the liver, spleen and lymph nodes were not palpable. Neither her own, nor her family history was contributory. Acute viral or secondary bacterial infection was suspected based on the following clinical laboratory findings upon admission: white blood cell (WBC) count, $11,780 / \mathrm{mm}^{3}$ (neutrophils $84.6 \%$ ), platelets, $15.4 \times 10^{4} /$ $\mathrm{mm}^{3}$ and C-reactive protein (CRP), 13.6 IU/L. However, we identified a bite with eschar on her left arm (Fig. 1), and learned that the patient frequently pursued outdoor activities. We tentatively diagnosed scrub typhus (tsutsugamushi disease), a common type of rickettsial disease caused by Orientia tsutsugamush (O. tsutsugamushi). However, despite the administration of minocycline hydrochloride (200 mg/day) with panipenem/betamipron (1 g/day), the fever persisted

\footnotetext{
${ }^{1}$ Nagasaki University Graduate School of Biomedical Sciences, Department of Molecular Microbiology and Immunology, the Second Department of Internal Medicine, Nagasaki University School of Medicine, Nagasaki, ${ }^{2}$ Kitakyushu City Yahata Hospital, Kitakyusyu and ${ }^{3}$ Miyazaki Prefectural Institute for Public Health and Environment, Miyazaki

Received for publication March 20, 2006; Accepted for publication August 10, 2006

Correspondence to Dr. Masafumi Seki, the Second Department of Internal Medicine, Nagasaki University School of Medicine, 1-7-1 Sakamoto, Nagasaki 852-8501
} 
(A)

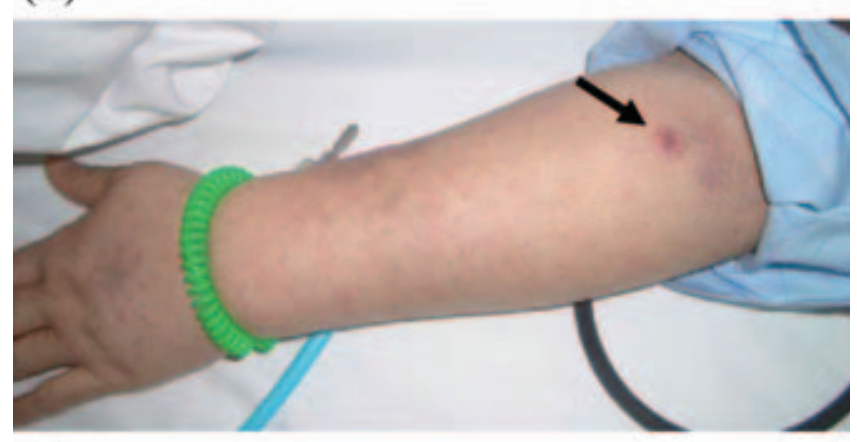

(B)

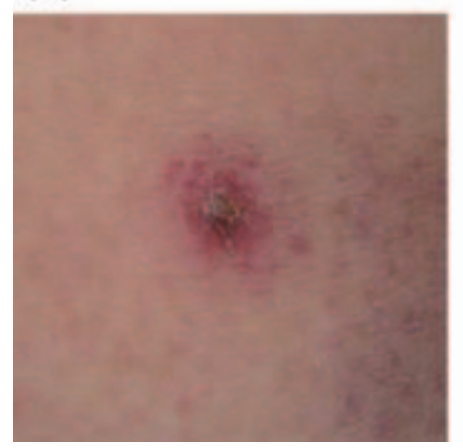

Figure 1. A tick bite eschar on admission of the patient. (A) The escher was found on her left arm (arrow). (B) Enlarged photo of the bite escher.

and clinical laboratory findings on May 10th (Clinical Day 10) revealed CRP, 23.6 IU/L and a platelet count of $7.6 \times 10^{4} /$ $\mathrm{mm}^{3}$, indicating deterioration (Fig. 2). Because we suspected other pathogens and DIC: disseminated intravascular coagulation, we replaced the minocycline hydrochloride with fluoroquinolone: ciprofloxacin hydrochloride (600 mg/day) and continued with the panipenem/betamipron. Her condition was obviously improved 3 days later and the fever decreased to $36.7^{\circ} \mathrm{C}$. Levels of WBC and CRP were also decreased to $8,600 / \mathrm{mm}^{3}$ and $9.7 \mathrm{IU} / \mathrm{L}$, respectively and on Clinical Day 15 , the platelet count increased to $15.3 \times 10^{4} /$ $\mathrm{mm}^{3}$. We discontinued the panipenem/betamipron on Clinical Day 15 and ciprofloxacin on Clinical Day18. She was discharged from the hospital on Clinical Day 20.

Serological tests (indirect immunofluorescence analysis) upon admission using antibodies were negative for $O$. tsutsugamushi (Karp, Kato, Gilliam, Kawasaki and Kuroki strain: data not shown) and $R$. japonica (YH strain), however, she became seropositive for $R$. japonica alone 20 days after admission (Fig. 2). Because of these results and clinical course, the patient was diagnosed as Japanese spotted fever, one of the spotted fever group rickettsial diseases.

\section{Discussion}

A patient with severe Japanese spotted fever was successfully treated with ciprofloxacin hydrochloride. We found a unique bite with eschar, and initially suspected scrub typhus,

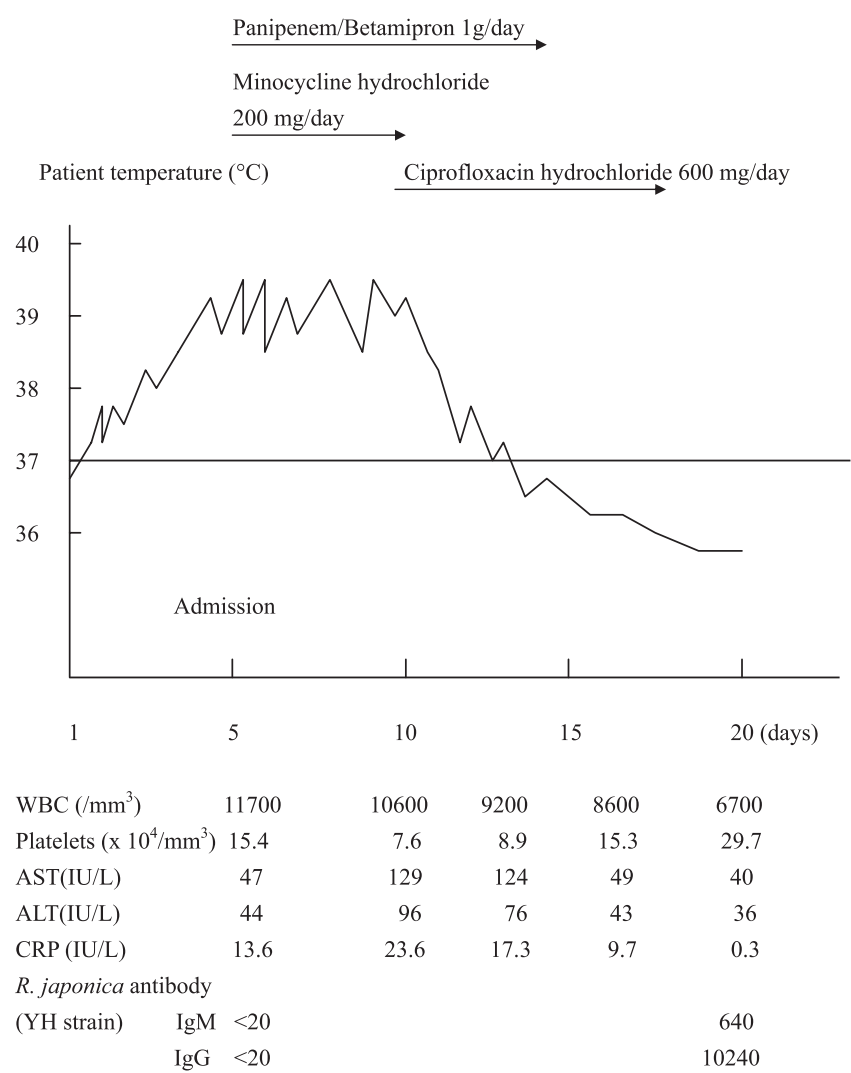

Figure 2. Clinical course of the patient.

a common rickettsial condition in Japan (1). However, minocycline hydrochloride, a tetracycline drug that is highly effective against not only scrub typhus (1), but also Japanese spotted fever (2), had no effect. We also suspected bacterial infection, including cat scratch disease by Bartonella henselae, however, we did not find any lymph node swellings and we did not detect any microorganisms or related antigens in the blood, urine or central spinal fluid. Urine antigen for Streptococcus pneumoniae and Legionella pneumophila was not detected (data not shown). Chest and abdominal X-rays and CT findings showed no abnormalities, and gastrointestinal and/or genitourinary co-infections by gram-negative bacteria which were usually resistant to tetracyclines, but effective to fluoroquinolones, were not found. In addition, no prevalent past history regarding food or drugs were found before admission.

Tetracyclines, fluoroquinolones, and macrolides are the most effective antibiotics against rickettsial strains in vitro (3), and no tetracycline-resistant $R$. japonica has been reported including this study. However, the spotted fever group rickettsiae, $R$. belli, and $R$. Canada are more resistant with josamycin, whereas typhus group rickettsiae are susceptible to all macrolides tested (3). On the other hand, doxycycline-resistant, but azithromycin-susceptible strains of O. tsutsugamushi were examined in vitro, and the shorter half-life of doxycycline (18-22 hr), compared with that of azithromycin (68 hr) was discussed as a reason of occasional failure of treatment by tetracyclines for scrub ty- 
(A)

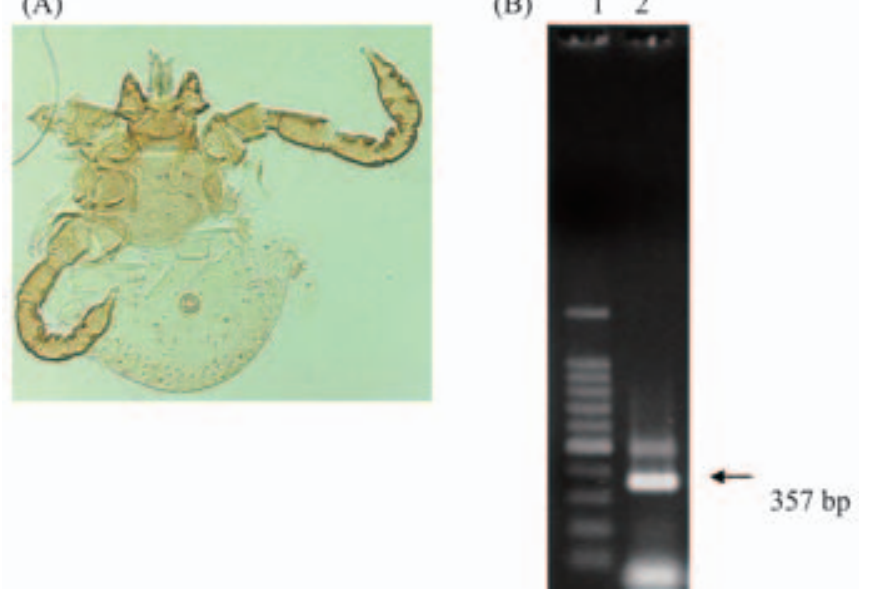

Figure 3. PCR method for $R$. japonica from the tick. (A) The tick: Larva of Haemaphysalis hystricis collected in the mountain where the patient was infected. (B) Detection of $\boldsymbol{R}$. japonica specific DNA from the tick Lane 1, 1-kb ladder marker; Lane 2, tick sample. 357 bp band (arrow) indicates the fragment specific for $R$. japonica.

pus (4)

The half-life of ciprofloxacin is very short $(2.6 \mathrm{hr})$, but there is higher penetration into tissue (ex. lung; ciprofloxacin (5) $4.9 \pm 2.0 \mathrm{microg} / \mathrm{g}$ vs minocycline (6): $2.9 \pm 1.4 \mathrm{mi}-$ crog $/ \mathrm{g})$ and cells $(7,8)$ may be a reason that ciprofloxacin is a more effective antibiotic than tetracyclines for bacteria which grow intracellularly, including rickettsiae. Furthermore, fluoroquinolones were suggested to be able to kill most, if not all, of the bacteria present in infected cells and are removed from cells without causing rapid bacterial regrowth in legionnaires diseases $(7,8)$.

In clinical case, Yagupsky and Gross described a child in Israel with spotted fever group rickettsioses was treated with a 3.5 day course of doxycycline and subsequently suffered a relapse of the disease, which indicates that the in vivo activity of doxycycline may be only rickettsiostatic (9).

Iwasaki et al. also reported that fluoroquinolones are required to eradicate the causative rickettsiae of Japanese spotted fever, regardless of inflammatory cytokine modulation by minocycline hydrochloride (10). In their case, the initial treatment with minocycline hydrochloride and methylpredni- solone was successful, however, the patient's fever relapsed and was resolved only after the addition of ciprofloxacin hydrochloride.

Rickettsiae are susceptible to fluoroquinolone, with MICs of 0.125 to $0.25 \mu \mathrm{g} / \mathrm{ml}$ for $R$. rickettsii and 0.25 to $0.5 \mu \mathrm{g} /$ $\mathrm{ml}$ for $R$. conorii (11). These data suggest that fluoroquinolone, rather than tetracyclines, might be sufficiently effective against Japanese spotted fever, so patients may require an additional administration of fluoroquinolones (12). Fluoroquinolones may be needed to completely eradicate the causative rickettsiae. Moreover, fluoroquinolones should be administered as soon as antibiotics are deemed ineffective and rickettsial diseases are strongly suspected, because some spotted fever-type rickettsial diseases including Japanese spotted fever, can be fatal $(1,2,13)$.

After the patient recovered, we searched for ticks, such as the vector of $R$. japonica on the mountain where the patient had been bitten on June 8 . We collected a total of 24 ticks, consisting of 2 genera and 5 species. We detected $R$. japonica-specific DNA in these ticks by the polymerase chain reaction (PCR) using specific primers $(13,14)$ for $R$. japonica. The DNA was specifically detected in the tick: Haemaphysalis hystricis (H. hystricis) larvae (Fig. 3). Genetic analysis revealed that this species is an arthropod reservoir for $R$. japonica $(15,16)$. The present patient may have been bitten by the tick, including $H$. hystricis harboring $R$. japonica.

We encountered severe Japanese spotted fever in Fukuoka prefecture. Most Japanese spotted fever cases have been reported from along the Pacific coast, as well as in southwestern and central Japan (12, 17-19). However, Japanese spotted fever has recently been diagnosed in patients from areas around the Japan Sea $(20,21)$ and in Korea $(22)$. The distribution of Japanese spotted fever may be spreading.

In conclusion, we described a case of severe Japanese spotted fever that was successfully treated by fluoroquinolone, but not minocycline. The pathogen was detected in ticks collected from the mountain where the patient had been recently bitten. Japanese spotted fever may become more prevalent. Careful examination and early appropriate antibiotic administration is a prerequisite to treat this condition.

\section{References}

1. Mandell GE, Benette JE, Dolin R. Principles and Practice of Infectious Diseases. 6th edition. Elsevier-Churchill Livingstone, Philadelphia, 2005: 2284-2318.

2. Kodama K, Senba T, Yamauchi H, Nomura T, Chikahira Y. Clinical study of Japanese spotted fever and its aggravating factors. J Infect Chemother 9: 83-87, 2003.

3. Rolain JM, Maurin M, Vestris G, Raoult D. In vitro susceptibilities of 27 rickettsiae to 13 antimicrobials. Antimicrob Agents Chemother 42: 1537-1541, 1998.

4. Strickman D, Sheer T, Salata K, et al. In vitro effectiveness of azithromycin against doxycycline-resistant and -susceptible strains of Rickettsia tsutsugamushi, etiologic agent of scrub typhus. Antimicrob Agents Chemother 39: 2406-2410, 1995.

5. Haraguchi S, Hioki M, Yamashita $K$, et al. Penetration of ciprofloxacin into pulmonary parenchyma in Japanese patients. Jpn J Clin Surg 67: 277-280, 2006 (in Japanese).

6. Watanabe A, Anzai Y, Niitsuuma K, Saito M, Yanase K, Nakamura M. Penetration of minocycline hydrochloride into lung tissue and sputum. Chemotherapy 47: 1-9, 2001.

7. Edelstein PH. Antimicrobial chemotherapy for legionnnaires dis- 
ease: time for change. Ann Intern Med 129: 328-330, 1998.

8. Higa F, Kusano N, Tateyama M, et al. Simplified quantitative assay system for measuring activities of drugs against intracellular Legionella pneumophila. J Clin Microbiol 36: 1392-1398, 1998.

9. Yagupsky P, Gross E. Relapse of Israeli spotted fever treated with doxycycline. Trans R Soc Trop Med Hyg 79: 139-140, 1985.

10. Iwasaki H, Mahara F, Takada N, Fujita H, Ueda T. Fulminant Japanese spotted fever associated with hypercytokinemia. J Clin Microbiol 39: 2341-2343, 2001.

11. Raoult D, Bres P, Drancourt M, Vestris G. In vitro susceptibilities of Coxiella burnetii, Rickettsia rickettsii, and Rickettsia conorii to the fluoroquinolone sparfloxacin. Antimicrob Agents Chemother 35: 88-91, 1991.

12. Mahara F. Japanese spotted fever: report of 31 cases and review of the literature. Emerg Infect Dis 3: 105-111, 1997.

13. Kodama K, Tokieda S, Yamauchi H, et al. Fulminant Japanese spotted fever definitively diagnosed by the polymerase chain reaction method. J Infect Chemother 8: 266-268, 2002.

14. Furuya Y, Katayama T, Yoshida Y, Kaiho I. Specific amplification of Rickettsia japonica DNA from clinical specimens by PCR. J Clin Microbiol 33: 487-489, 1995.

15. Fournier PE, Fujita H, Takada N, Raoult D. Genetic identification of rickettsiae isolated from ticks in Japan. J Clin Microbiol 40:
2176-2181, 2002.

16. Noda S, Yamamoto S, Honda T. Detection of japanese spotted fever rickettsiae DNA from ixodid ticks and seasonal occurrence of ixodid tick population on the Osumi Peninsula, Kagoshima Prefecture. Med Entomol Zool 55: 21-28, 2004.

17. Kaiho I, Tokieda M, Ohtawara M, Uchiyama T, Uchida T. Occurrence of rickettsiosis of spotted fever group in Chiba Prefecture of Japan. Jpn J Med Sci Biol 41: 69-71, 1988.

18. Chiya S, Takahashi N, Yasuoka T, Komatsu T, Suzuki H. Japanese spotted fever cases in Kochi prefecture. Jpn J Infect Dis 53: 2729, 2000.

19. Takao S, Kawada Y, Ogawa M, et al. The first reported case of Japanese spotted fever in Hiroshima Prefecture, Japan. Jpn J Infect Dis 53: 216-217, 2000.

20. Itagaki A, Matsuda Y, Hoshina K. Japanese spotted fever in Shimane Prefecture: outbreak and place of infection. Jpn J Infect Dis 53: 73-74, 2000.

21. Noji Y, Takada N, Ishiguro F, et al. The first reported case of spotted fever in Fukui Prefecture, the northern part of central Japan. Jpn J Infect Dis 58: 112-114, 2005.

22. Jang WJ, Kim J, Choi YJ, et al. First serologic evidence of human spotted fever group rickettsiosis in Korea. J Clin Microbiol 42: 2310-2314, 2004.

(C) 2006 The Japanese Society of Internal Medicine http://www.naika.or.jp/imindex.html 